\title{
Induced parity-odd effective action for a Dirac field on $\mathcal{S}^{2} \times \mathcal{S}^{1}$
}

\author{
C. D. Fosco ${ }^{a}$ and F. A. Schaposnik ${ }^{b *}$ \\ ${ }^{a}$ Centro Atómico Bariloche and Instituto Balseiro, \\ Comisióon Nacional de Energía Atóomica, 8400 Bariloche, Argentina \\ ${ }^{b}$ Departamento de Física, Universidad Nacional de La Plata \\ Instituto de Física La Plata-CONICET \\ C.C. 67, 1900 La Plata, Argentina
}

September 23, 2018

\begin{abstract}
We evaluate the parity-odd part of the effective action due to massive Dirac fermions on a $\mathcal{S}^{2} \times \mathcal{S}^{1}$ manifold, minimally coupled to an external Abelian gauge field. We do that for a special class of gauge-field configurations, which is specially suitable to the study of the behaviour of the fermionic determinant under large gauge field configurations, which are allowed by the space-time geometry.
\end{abstract}

\section{Introduction}

Physical systems involving topological quantum matter (see [1] and reference therein) have recently aroused much attention and attracted intense theoretical research, in part because of the existence of dualities in their effective field theory model descriptions. The latter usually involve ChernSimons (CS) matter theories coupled to scalars, and similar theories coupled to fermions. Originally discussed in [2]-[4] these field theoretical investigations were extended in several directions [5]. In those models, two out of

${ }^{*}$ Associated with CICBA 
the three spatial dimensions of the real material are compactified to the $\mathcal{S}^{2}$ sphere, which is meant to describe a boundary (the third spatial coordinate is assumed to have a finite extension). Finally, in order to account for finitetemperature effects, the remaining, Euclidean time coordinate is an $\mathcal{S}^{1}$ circle of circumference $\beta=T^{-1}, T$ denoting the temperature (we adopt units such that Boltzmann's constant $k_{B} \equiv 1$ ).

In this way, phenomena which take place on the boundary of topological insulators or superconductors can be studied using models with bosons or fermions, coupled to external and dynamical gauge fields, with a CS action for the former and BF terms for their interaction.

Based on the previous motivation, systems described by actions defined on a manifold $\mathcal{M}=\mathcal{S}^{2} \times \mathcal{S}^{1}$, involving bosonic or fermionic matter minimally coupled to an external gauge field, have been studied. This has lead us to consider, in this paper, a particularly interesting object, namely, the effective action $\Gamma[A]$ for a massive Dirac field on precisely that kind of spacetime manifold.

It is a well-known property that $\Gamma[A]$, for Dirac fermions in $d=3$ (either Euclidean or Minkowskian) spacetime dimensions, contains a parity and time-reversal breaking term $\Gamma_{\text {odd }}[A]$. This is a reflection of the breaking of those symmetries; explicitly, when there is a finite fermion mass $m$ at the classical level, or implicitly by quantum effects, due to the (unavoidable) regularization. The main difference between those two contributions, besides their different origins, is that the one due to the regularization is local and independent of $m$, while the one corresponding to the explicit introduction of a mass term is local, and proportional to the CS action, only when $m$ tends to infinity 1. The structure of the mass-dependent, parity-odd term in the effective action at finite temperature cannot be determined just by symmetry considerations, but requires an explicit computation 2. The anomalous and the mass-dependent contributions to $\Gamma_{\text {odd }}[A]$ will be termed $\Gamma_{\text {odd }}^{(0)}[A]$ and $\Gamma_{\text {odd }}[A, m]$, respectively, so that

$$
\Gamma_{\text {odd }}[A]=\Gamma_{\text {odd }}^{(0)}[A]+\Gamma_{\text {odd }}[A, m] .
$$

The regularization procedure produces a global sign ambiguity in $\Gamma_{\text {odd }}^{(0)}[A]$. Indeed, within the $\zeta$-function regularization approach, this ambiguity is associated to the choice of a contour when defining the complex powers of the Dirac operator on odd-dimensional manifolds [6] and is independent of $m$.

\footnotetext{
${ }^{1}$ Or, equivalently, when keeping the leading terms in an expansion in derivatives.

${ }^{2}$ Let us note that under certain special conditions, out of our scope, a regularization involving the $\eta[A]$ invariant should be consider [2].
} 
Using this approach for fermions coupled to a $U(1)$ gauge field background in 3-dimensional Euclidean space, with the effective action defined by

$$
\exp (-\Gamma[A]) \equiv \int D \bar{\psi} D \psi \exp \left(\int_{\mathbb{R}^{3}} \bar{\psi}(\not \partial+i \not A+m) \psi\right)
$$

the result for the anomalous part has been shown to be [6]:

$$
\Gamma_{\text {odd }}^{(0)}[A]= \pm \frac{i}{2} S_{C S}[A]
$$

where

$$
S_{C S}=\frac{1}{4 \pi} \int d^{3} x \varepsilon^{\mu \nu \alpha} A_{\mu} \partial_{\nu} A_{\alpha} .
$$

Regarding the mass-dependent part of the parity-odd effective action $\Gamma_{\text {odd }}[A, m]$ one consistently obtains, either by using the $\zeta$-function approach or a derivative expansion for the external gauge field, the result:

$$
\Gamma_{\text {odd }}[A, m]=\frac{i}{2} \frac{m}{|m|} S_{C S}[A]+\mathcal{O}\left(m^{-2}\right) .
$$

Note that, by an appropriate choice of signs, one can cancel the $1 / 2$ factor in $\Gamma_{\text {odd }}[A]$ leading to an odd-parity effective action which is gauge invariant even for large gauge transformations. It is important to realize that the leading term in a mass expansion is the most relevant one when considering topological properties, like invariance under those large transformations.

In other regularization schemes, the same phenomenon must and does manifest itself; for instance, in the Pauli-Villars approach, the breaking may be traced back to the presence of a regulator, a spinorial field with a Diraclike action, but quantized using Bose-Einstein statistics. The sign of the mass of the regulator is not determined by the regularization. Removing the regularization at the end of the calculations amounts, in this case, to letting the regulator mass tend to infinity. This process yields a finite limit, the CS action, but with an overall sign which depends on the sign of the regulator mass.

It is the aim of this paper to evaluate the mass-dependent term $\Gamma_{\text {odd }}[A, m]$ for massive Dirac fermions on the manifold $\mathcal{M}=\mathcal{S}^{2} \times \mathcal{S}^{1}$. The anomalous part does indeed exist, and it is part of $\Gamma_{o d d}[A]$, but is has a well-known form: it is the integral of the CS 3 -form on $\mathcal{M}$, with the same coefficient as in the $T=0$ case.

This paper is organized as follows: in Section 2, we briefly review known results for the effective action corresponding to a massive Dirac fermion on the $\mathbb{R}^{2} \times \mathcal{S}^{1}$ manifold. Based on this approach, in Sect. 3 we consider the case of $\mathcal{S}^{2} \times \mathcal{S}^{1}$. In Section 4 we present a discussion of our results. 


\section{Massive Dirac fermions at finite temperature}

The study the effective action for a Dirac field in $2+1$ dimensions at finite temperature, in the Matsubara formalism one should regard the field as living on Euclidean $\mathbb{R}^{2} \times \mathcal{S}^{1}$ space. In this case, gauge invariance under large gauge transformations associated to the $\mathcal{S}^{1}$ "time" is spoiled at any finite order in a perturbative calculation in powers of the gauge coupling constant. That invariance under large gauge transformations can, however, be rescued, if one follows a non-perturbative approach, like the ones presented in [9]-[11].

The key idea in refs. [10]-11] was to first write the gauge invariant parityodd part $\Gamma_{\text {odd }}$ of the effective action $\Gamma$ due to the fermions in the presence of an external gauge field, to then reduce the problem to the calculation of a set of Fujikawa Jacobians in $R^{2}$ space and finally to obtain $\Gamma_{\text {odd }}$ by summing over Matsubara modes associated to the discrete Fourier transformations in the "time variable".

The above "reduction" mechanism whereby the problem was essentially reduced to a collection of $1+1$ systems was implemented for a certain class of gauge field configurations,

$$
A_{i}=A_{i}(x), \quad A_{3}=A_{3}(\tau),
$$

where we have adopted the convention (to be followed in the rest of this paper) to denote by $x$ just the two spatial coordinates, namely $x=\left(x_{1}, x_{2}\right)$. We will also assume that indices from the middle of the Latin alphabet will run from 1 to 2, while if they belong to the Greek one, their range is from 1 to 3 . The third Euclidean coordinate will be alternatively denoted as $\tau$ or $x_{3}$.

Note that (6) may be regarded as a gauge-fixed version of the gaugeinvariant conditions

$$
F_{3 i}=0, \quad F_{i j}=F_{i j}(x),
$$

which correspond to field configurations which, if the gauge field were determined by a Maxwell Lagrangian in $2+1$ dimensions, would be those of pure magnetostatics. Namely, configurations produced by a vanishing charge density and a solenoidal time-independent current $J_{i}(x)$ which determines $F_{i j}$ via Ampère's Law:

$$
\partial_{j} F_{j i}(x)=-J_{i}(x) .
$$

Since $\partial_{i} J_{i}=0$, we may write it as the curl of a pseudoscalar $\zeta(x)$, namely: $J_{i}(x)=\epsilon_{i j} \partial_{j} \zeta(x)$. In terms of the magnetic field $B(x)=\frac{1}{2} \epsilon_{i j} F_{i j}(x)$, we see that (8) becomes:

$$
B(x)=F_{12}(x)=\zeta(x) .
$$


Let us now describe the finite temperature calculation. In the pathintegral framework, one works with an Euclidean time in the interval $[0, \beta]$, imposing anti-periodic boundary conditions to the fermions in that interval. Hence the manifold becomes $\mathbb{R}^{2} \times \mathcal{S}^{1}$ and then one sees that invariance under large gauge transformations associated to the $\mathcal{S}^{1}$ domain is spoiled at any finite order in the gauge coupling constant (see [12] and references therein). This fact has caused some confusion regarding the CS coefficient discreteness condition, since the coefficient of the CS action would then satisfy a rather unphysical $\beta$-dependent quantization condition in conflict with large gauge invariance of the effective action.

The problem has been solved by following non-perturbative approaches. We shall briefly describe here the procedure followed in refs. [10]-[11] to perform such calculation. The main idea was to reduce the problem to the calculation of a set of Fujikawa Jacobians on an $\mathbb{R}^{2}$ space, and then to obtain the complete partition function by summing over Matsubara modes associated to the Fourier transformations on $\mathcal{S}^{1}$.

The mass-dependent 3 part of $\Gamma_{\text {odd }}$ may be obtained by using the expression:

$$
\Gamma_{\text {odd }}[A, m]=\frac{1}{2}(\Gamma[A, m]-\Gamma[A,-m]),
$$

for a system defined by the action

$$
S=\int_{0}^{\beta} d \tau \int_{\mathbb{R}^{2}} \bar{\psi}(\not \partial+i \not A+m) \psi .
$$

The result has been shown to be:

$$
\begin{aligned}
\Gamma_{\text {odd }}[A, m]= & \frac{i}{2 \pi} \frac{m}{|m|} \arctan \left[\tanh \left(\frac{\beta|m|}{2}\right) \times \tan \left(\frac{1}{2} \int_{0}^{\beta} d \tau A_{3}(\tau)\right)\right] \\
& \times \int_{\mathbb{R}^{2}} d^{2} x F_{12}
\end{aligned}
$$

This result, obtained for the class of configurations explicited in (6), has the correct zero-temperature limit:

$$
\lim _{\beta \rightarrow \infty} \Gamma_{\text {odd }}^{I}[A]=\frac{i}{2} \frac{m}{|m|} \frac{1}{4 \pi} \int d^{3} x \varepsilon^{\mu \nu \alpha} A_{\mu} \partial_{\nu} A_{\alpha}=\frac{i}{2} \frac{m}{|m|} S_{C S} .
$$

The result in Eq.(13) is not invariant by itself under large gauge transformations, because of the $1 / 2$ factor affecting the CS term. The same happens

\footnotetext{
${ }^{3}$ Note that only the mass-dependent part of $\Gamma_{\text {odd }}$ may depend non-trivially on $\beta$.
} 
in the finite temperature result as given by eq.(12). Indeed, if one makes a large gauge transformation $\Omega_{n}\left(x_{1}, x_{2}, \tau\right)$ with $n=2 p+1$ that winds an arbitrary number $n$ of times around cyclic time direction,

$$
\Omega_{n}(\beta, x)=\Omega_{n}(0, x)+2 \pi n, \quad n \in \mathbb{Z}
$$

the argument in the tangent of eq. (12) is shifted by $(2 p+1) \pi$. Although the tangent is not sensitive to such a change, one has to keep track of it by shifting the branch used for the arctan definition so that $\Gamma_{\text {odd }}[A]$ changes by the addition of $i n \pi$ under a large gauge transformation and for odd $n=2 k+1$ one should correct this with an appropriate gauge invariant regularization.

Note that this gauge non-invariance can be compensated by a judicious choice of the sign in the anomalous term, which has an analogous behaviour under the same transformations.

Some properties of $\Gamma_{\text {odd }}[A, m]$ may also be studies by considering its contribution $\theta_{\text {odd }}[A, m]$ to the phase of the fermionic determinant,

$$
e^{i \theta_{\text {odd }}[A, m]} \equiv e^{-\Gamma_{\text {odd }}[A, m]}
$$

where the angle $\theta_{\text {odd }}[A, m]$ can be written as follows:

$$
\theta_{\text {odd }}[A, m]=n_{B} \arg (z)
$$

where

$$
z \equiv \cos \left(\frac{1}{2} \int_{0}^{\beta} d \tau\left[A_{3}(\tau)+i m\right]\right)
$$

and $n_{B} \equiv \frac{1}{2 \pi} \int_{\mathbb{R}^{2}} d^{2} x F_{12}$, which is quantized for non-singular configurations, namely, $n_{B} \in \mathbb{Z}$.

We conclude this review Section commenting the fact that the previous result for $\Gamma_{\text {odd }}$ may be generalized to a slightly more general class of configurations. Indeed, having in mind the fact that the ones considered before were purely magnetostatic ones, we can think about the generalization to the case of having static magnetic plus electric fields:

$$
F_{3 i}=F_{3 i}(x), \quad F_{i j}=F_{i j}(x) .
$$

In this case, the only change one has to introduce in the derivation of the previous case is the fact that the anomalous Jacobian corresponds to a spacedependent phase, and the result becomes the straightforward generalization:

$$
\Gamma_{\text {odd }}[A, m]=i \frac{m}{|m|} \int_{\mathbb{R}^{2}} d^{2} x \mathcal{L}[A, m, \beta]
$$


where

$$
\mathcal{L}[A, m, \beta]=\frac{1}{2 \pi} \arctan \left[\tanh \left(\frac{\beta|m|}{2}\right) \tan \left(\frac{1}{2} \int_{0}^{\beta} d \tau A_{3}(x, \tau)\right)\right] F_{12}(x) .
$$

\section{Massive Dirac field on $\mathcal{S}^{2} \times \mathcal{S}^{1}$ : the determinant}

We consider now a massive Dirac field $\psi, \bar{\psi}$, living on the manifold $\mathcal{M} \equiv S^{2} \times S^{1}$, where $S^{1}$ is a periodic (imaginary) time coordinate, while $S^{2}$ denotes a spatial sphere of radius $R$.

The manifold $\mathcal{M}$ is equipped with the product metric, namely

$$
d s^{2}=(d \tau)^{2}+g_{i j}(x) d x^{i} d x^{j},
$$

which corresponds to a particular case of static space-time.

For a field defined on $\mathcal{M}$, in the presence of an external Abelian gauge field $A$, we are interested in evaluating the imaginary part of the Euclidean effective action, $\Gamma(A, m)$, where:

$$
e^{-\Gamma(A, m)}=\int \mathcal{D} \psi \mathcal{D} \bar{\psi} e^{-\mathcal{S}(\bar{\psi}, \psi, A, m)},
$$

where the Dirac field is again assumed to be minimally coupled to an Abelian gauge field $A_{\mu}(x)$, so that the action $\mathcal{S}$ is defined as follows:

$$
\mathcal{S}(\bar{\psi}, \psi, A, m)=\int_{\mathcal{M}} d^{3} x \sqrt{g} \bar{\psi}(x)\left(\mathcal{D}_{\mathcal{M}}+m\right) \psi(x),
$$

where $\mathcal{D}_{\mathcal{M}}$ denotes the Dirac operator corresponding to $\mathcal{M}$ and coupling to the gauge field $A_{\mu}$.

Some objects appearing in the action involve the geometry of $\mathcal{M}$ in a more specific way: $g \equiv \operatorname{det}\left(g_{\mu \nu}\right)=\operatorname{det}\left(g_{i j}\right)$, where $g_{\mu \nu}$ may be represented in matrix form:

$$
\left(g_{\mu \nu}\right)=\left(\begin{array}{ccc}
g_{11}(\mathbf{x}) & g_{12}(\mathbf{x}) & 0 \\
g_{21}(\mathbf{x}) & g_{22}(\mathbf{x}) & 0 \\
0 & 0 & 1
\end{array}\right) .
$$

Denoting by $x^{i} \rightarrow \mathbf{r}\left(x^{1}, x^{2}\right)$ a parametrization of the surface $\mathcal{S}^{2}$ :

$$
\mathbf{r}\left(x^{1}, x^{2}\right) \in \mathbb{R}^{3}, \quad \mathbf{r}\left(x^{1}, x^{2}\right) \cdot \mathbf{r}\left(x^{1}, x^{2}\right)=R^{2}
$$


(where the dot denotes the Euclidean scalar product in $\mathbb{R}^{3}$ ), we have the spatial components $g_{i j}$ of the induced metric tensor:

$$
g_{i j}=\partial_{i} \mathbf{r} \cdot \partial_{j} \mathbf{r} .
$$

Besides, note that, because of the special form of the metric tensor above, the only non-trivial vierbeins are the two corresponding to $S^{2}$ (they are moreover time-independent).

Following the strategy of refs. [10]-[11] described above, we shall consider in this note the case in which the parity odd part of the effective action is calculated for fermions on $\mathcal{S}^{2} \times \mathcal{S}^{1}$.

\subsection{Going from $\mathcal{S}^{2} \times \mathcal{S}^{1}$ to $\mathcal{S}^{2}$}

For the magnetostatic configurations, we see that the only $\tau$ dependence of the Dirac operator comes from $A_{3}$. This dependence can, however, be eliminated by a redefinition of the integrated fermion fields. The set of allowed gauge transformations in the imaginary time formalism is defined in the usual way:

$$
\begin{aligned}
\psi(\tau, x) & \rightarrow \exp [-i \Omega(\tau, x)] \psi(\tau, x) \\
\bar{\psi}(\tau, x) & \rightarrow \exp [i \Omega(\tau, x)] \bar{\psi}(\tau, x) \\
A_{\mu}(\tau, x) & \rightarrow A_{\mu}(\tau, x)+\partial_{\mu} \Omega(\tau, x)
\end{aligned}
$$

where $\Omega(\tau, x)$ is a differentiable function vanishing at spatial infinity $|x| \rightarrow \infty$, and whose time boundary conditions are chosen in order not to affect the fields' boundary conditions. It turns out that $\Omega(\tau, x)$ can wind an arbitrary number of times around the cyclic time dimension,

$$
\Omega(\tau, x)=\Omega(\tau, x)+2 \pi n
$$

where $n$ is an integer which labels the homotopy class of the gauge transformation.

$Z[A]$ must be gauge invariant so that we can compute it for time independent gauge fields since one can always perform a gauge transformation $A_{\mu}^{\prime}=A_{\mu}+\partial_{\mu} \Lambda$ so that the $A_{\mu}^{\prime}$ is time-independent. For the particular set of configurations (6) such a transformation renders $A_{3}^{\prime}$ constant. We see that there is a family of $\Omega^{\prime} s$ achieving this while respecting the boundary conditions (28),

$$
\Omega(\tau)=-\int_{0}^{\tau} d \tilde{\tau} A_{3}(\tilde{\tau})+\frac{1}{\beta}\left(\int_{0}^{\beta} d \tilde{\tau} A_{3}(\tilde{\tau})+2 \pi n\right) \tau .
$$


The freedom to choose $n$ could be used to further restrict the values of the constant $A_{3}^{\prime}$ to a finite interval. In this sense, the value of the constant in such an interval is the only "essential" i.e., gauge invariant, $A_{3}$-dependent information contained in the configurations (6), describing the gauge connection holonomy $\exp \left(i \int_{0}^{\beta} d \tilde{\tau} A_{3}(\tilde{\tau})\right)$.

Let us, for the time being, disregard the case of large gauge transformations (i.e., we restrict to the case $n=0$ in (28)) in order to avoid any assumption about large gauge invariance of the fermionic measure in (22) and then discuss this issue on the final results. Thus, the constant field $A_{3}^{\prime}$ simply takes the mean value of $A_{3}(\tau), \tilde{A}_{3}=\frac{1}{\beta} \int_{0}^{\beta} d \tilde{\tau} A_{3}(\tau)$. Concerning the spatial components $A_{i}$, they remain $\tau$ independent after this transformation.

At this point we proceed to perform a Fourier transformation in the fermion variables,

$$
\begin{aligned}
\psi(\tau, x) & =\frac{1}{\beta} \sum_{n=-\infty}^{n=\infty} e^{i \omega_{n} \tau} \psi_{n}(x), \\
\bar{\psi}(\tau, x) & =\frac{1}{\beta} \sum_{n=-\infty}^{n=\infty} e^{-i \omega_{n} \tau} \psi_{n}(x),
\end{aligned}
$$

where $\omega_{n}=(2 n+1) \pi / \beta$ is the usual Matsubara frequency for the case of fermion fields. With this the Dirac action takes the form of an infinite series of decoupled two-dimensional Euclidean Dirac actions

$$
S_{D}=\frac{1}{\beta} \sum_{n=-\infty}^{n=\infty} \int_{S^{2}} d^{2} x \sqrt{g} \bar{\psi}_{n}(x)\left(\not D_{2}+M+i \gamma_{3}\left(\omega_{n}+\tilde{A}_{3}\right)\right) \psi_{n}(x) .
$$

Here, $\not D_{2}$ denotes the 2-dimensional Dirac operator acting on fermions living in the sphere $\mathcal{S}^{2}$; it is given by

$$
\not D_{2}=\gamma_{a} e_{a}^{k}\left(\partial_{k}+\frac{1}{4} \gamma_{c} \gamma_{d} \omega_{k c d}+i A_{k}(x)\right)
$$

Parametrizing $\mathcal{S}^{2}$ in terms of angular coordinates $\left(x^{1}, x^{2}\right)=(\theta, \varphi)$, the induced metric becomes $\operatorname{diag} g_{i j}=\left(R^{2}, R^{2} \sin ^{2} \theta\right)$, while the vierbeins $e_{i}^{a}$ $(a, b=1,2)$ adopt the form:

$$
\begin{array}{ll}
e_{\theta}^{1}=R \cos \varphi, & e_{\varphi}^{1}=-R \sin \varphi \sin \theta \\
e_{\theta}^{2}=R \sin \varphi, & e_{\varphi}^{2}=R \cos \varphi \sin \theta .
\end{array}
$$


The only non-trivial component of the spin connection is $\omega_{\varphi 12}=(1-\cos \theta)$ so that

$$
\not D_{2}=\gamma_{a} e_{a}^{k}\left(\partial_{k}+\frac{1}{4} i \delta_{k}^{\varphi} \gamma_{3}(1-\cos \theta)+i A_{k}(x)\right) \text {. }
$$

Thus, the action $S_{D}$ in (31) can be written in the form

$$
S_{D}=\frac{1}{\beta} \sum_{n=-\infty}^{n=\infty} \int_{S^{2}} d^{2} x \sqrt{g} \bar{\psi}_{n}(x)\left(\not D_{2}+\rho_{n} \exp \left(i \gamma_{3} \phi_{n}\right)\right) \psi_{n}(x),
$$

where

$$
\rho_{n}=\left(M^{2}+\left(\omega_{n}+\tilde{A}_{3}\right)^{2}\right)^{1 / 2}, \quad \phi_{n}=\arctan \left(\frac{\omega_{n}+\tilde{A}_{3}}{M}\right) .
$$

Again, the path-integral measure factorizes:

$$
\int D \bar{\psi} D \psi=\prod_{n=-\infty}^{n=\infty} D \bar{\psi}_{n}(x) D \psi_{n}(x)
$$

so that the partition function becomes a product

$$
\begin{aligned}
e^{-\Gamma[A, m]} & =\prod_{n=-\infty}^{n=\infty} D \psi_{n} D \psi_{n} e^{-\int_{\mathcal{S}_{2}} d^{2} x \sqrt{g} \bar{\psi}_{n}(x)\left(\not D_{2}+\rho_{n} e^{i \gamma_{3} \phi_{n}}\right) \psi_{n}(x)} \\
& \equiv \prod_{n=-\infty}^{n=\infty} \operatorname{det}\left(\not D_{2}+\rho_{n} e^{i \gamma_{3} \phi_{n}}\right)
\end{aligned}
$$

Now one can make in each pair of integrals in the infinite product the change of variables,

$$
\psi_{n}(x)=\exp \left[\left(-i \phi_{n} / 2\right) \gamma_{3}\right] \psi_{n}^{\prime}(x), \quad \bar{\psi}_{n}(x)=\bar{\psi}_{n}^{\prime}(x) \exp \left[\left(-i \phi_{n} / 2\right) \gamma_{3}\right],
$$

which can be seen as a a two-dimensional "chiral rotation" with $\gamma_{3}$ identified as the $d=2 \gamma_{5}$ matrix. Such changes eliminates the phases $\phi_{n}$ in the Dirac operators at the cost of a nontrivial Fujikawa Jacobians $J_{n}$ associated to the chiral anomaly. Indeed, each Dirac operator determinant in (38) is a product of eigenvalues that grow with no bound thus requiring a regularization. One can use for example the heat-kernel regularization which consists in introducing the identity

$$
I=\lim _{\Lambda \rightarrow \infty} \exp \left(-\left(\not D_{2}\right)^{2} / \Lambda^{2}\right)
$$


using as regulating operator the one in the action that ensures gauge invariance. One can easily see that the presence of the spin connection contribution does not change the result of the Jacobian with respect to the flat space case so that one finds

$$
J_{n}=\exp \left(-i \frac{e \phi_{n}}{2 \pi} \int_{\mathcal{S}^{2}} d^{2} x \varepsilon_{j k} \partial_{j} A_{k}\right)
$$

so that finally one ends up with

$$
\exp (-\Gamma[A, m])=\prod_{n=-\infty}^{n=\infty} J_{n} \operatorname{det}\left(\not D_{2}+\rho_{n}\right)
$$

Since the determinants in this formula do not depend on $m$ and our method to calculate $\Gamma_{\text {odd }}[A, m]$ consists in substracting the positive and negative mass results, they do not contribute to the parity odd effective action. In contrast, $\phi_{n}$ evidently depends on the sign of the mass. We then have

$$
\Gamma_{\text {odd }}=-\sum_{-\infty}^{\infty} \log J_{n}=\frac{i}{2 \pi} \sum_{-\infty}^{\infty} \phi_{n} \int_{\mathcal{S}^{2}} d^{2} x \varepsilon_{j k} \partial_{j} A_{k} .
$$

Performing the summation of the series, we obtain

$$
\Gamma_{\text {odd }}=\frac{i}{2 \pi} \frac{m}{|m|} \arctan \left(\tanh \left(\frac{\beta|m|}{2}\right) \tan \frac{1}{2} \int_{0}^{\beta} d \tau A_{3}(\tau)\right) \times \int_{\mathcal{S}^{2}} d^{2} x \varepsilon_{j k} \partial_{j} A_{k},
$$

which is the main result of this note.

Note that, again, invariance of the full effective action under large gauge transformations may be achieved, by taking into account the anomalous term with the proper sign. Equation (44) not only does have the proper zerotemperature limit, but it also produces a $\theta$-vacua term in the opposite, hightemperature limit, an object known to be present in the massive Schwinger model [13]. Indeed, we see that, when $\beta m$ tends to zero, the leading nontrivial term is

$$
\Gamma_{\text {odd }}[A, m] \sim i \theta \int_{\mathcal{S}^{2}} d^{2} x \varepsilon_{j k} \partial_{j} A_{k},
$$

with the dimensionless angle $\theta \equiv \frac{\beta^{2} m \tilde{A}_{3}}{8 \pi}$. This behaviour is to be expected, since one should obtain a result corresponding to massive fermions in 2 dimensions. 


\section{Discussion}

We have computed the exact mass-dependent parity-odd effective action for fermions coupled to a $U(1)$ gauge field for a $\mathcal{S}^{2} \times \mathcal{S}^{1}$ manifold. Once the standard anomalous $\Gamma^{(0)}[A]=\frac{1}{2} S_{C S}[A]$ contribution is incorporated, gauge invariance holds even when large gauge transformations are considered. Interestingly enough, the results coincides with the one obtained in [10] since the inclusion of the spin connection in the Dirac operator does not affect the result.

Concerning the extension to the case of non-Abelian gauge symmetries, the issue of large gauge invariance in non-Abelian finite temperature effective actions for the case of an $\mathbb{R}^{2} \times \mathcal{S}^{1}$ manifold has been discussed in [14], and the explicit calculation of $\Gamma_{\text {odd }}[A]$ for fermions in the fundamental representation of $S U(N)$ presented in [11] so that we expect that the extension of the present analysis to the non-Abelian case will follow the same steps for the $\mathcal{S}^{2} \times \mathcal{S}^{1}$ manifold and lead to similar results as those presented here.

Note that the flux quantization condition, namely, that $\frac{\Phi_{B}}{2 \pi} \in \mathbb{Z}$ can be altered when one considers a path integral involving the insertion of matter field operators with half-integer spins, since now the usual argument leading to Dirac's quantization condition has to be applied to a field which is doublevalued, i.e., changes sign under $2 \pi$ rotations. Thus, one can expect the value of $\frac{\Phi_{B}}{2 \pi}$ to be a half-integer in some cases. Of course, a full consideration has to be made of the contributions of the gauge field action itself, when that field is dynamical, since it can also put constraints on the allowed values of the flux (for example, by requiring finite energies). Even in a situation when the flux is a half integer times $2 \pi$, large gauge invariance may be restored by the presence of the anomalous term with the proper sign and coefficient. In the context of Pauli-Villars regularization, that would correspond to having three, rather than one, regulator fields. This number of regulators, is not required in the calculation of the fermion loop (where one is sufficient); rather, it is needed in order to regulate diagrams with operator insertions, where the superficial degree of divergence is increased.

It is important to note that the reduction of the $2+1$ dimensional system to a collection of 2-dimensional ones, for the case of a static metric like the one in (21), can certainly be generalized to spatial manifolds different from the sphere. Indeed, the main properties we relied upon to achieve the reduction still hold true, in particular, the fact that the vierbeins are effectively two dimensional, they do not mix with the time-like coordinate.

Let us conclude by pointing out that the knowledge of the fermionic determinant is an essential ingredient in the path-integral approach to bosoniza- 
tion in $d \geq 2$ dimensions, as developed in refs.[15]-[16]. Indeed, in 3 spacetime dimensions, the fermionic action and its $\bar{\psi} \gamma^{\mu} \psi$ current become related, by a duality transformations, to an effective low energy Chern-Simons action and a conserved dual current on the bosonic side. Thus, we believe, the issue of having non perturbative results for the fermionic determinant may be relevant to the correct implementation of the bosonization/duality programs at a finite temperature, within the context of condensed matter physics. We have shown that the fermionic determinant leads to a non-local gauge action which reduces to a CS action solely in the $T \rightarrow 0$ limit and this takes place both for the spatial coordinates taking values in $\mathbb{R}^{2}$ or $\mathcal{S}^{2}$. Moreover, it becomes a $\theta$-vacua term when the opposite, high temperature limit is considered.

Finally, note also that the program of extending the study of dualities to different manifolds could also be considered in higher dimensions: indeed, in $d>3$ dimensions, it has been shown that the dual current involves a $d-2$ Kalb-Ramond field [17]. Regarding the massless fermion case, different dualities connect the fermionic theory with vector and scalar fields theories [18] and one can even consider the case in which fermions are coupled to a gravitational background [19]. We hope to report on the result of that extension, as well as on the consequences for condensed matter applications, in future works.

\section{ACKNOWLEDGEMENTS}

This work was supported by ANPCyT, CICBA, CONICET, UBA, UNCuyo and UNLP.

\section{References}

[1] M. Z. Hasan and C. L. Kane, Rev. Mod. Phys. 82 (2010) 3045.

[2] N. Seiberg, T. Senthil, C. Wang and E. Witten, Annals Phys. 374 (2016) 395.

[3] A. Karch and D. Tong, Phys. Rev. X 6 (2016) no.3, 031043

[4] J. Murugan and H. Nastase, arXiv:1606.01912 [hep-th].

[5] P. S. Hsin and N. Seiberg, JHEP 1609 (2016) 095; Đ. Radicevic, D. Tong and C. Turner, JHEP 1612 (2016) 067; A. Karch, B. Robinson and D. Tong, JHEP 1701 (2017) 017; A. Cappelli, E. Randellini and J. Sisti, arXiv:1612.05212 [cond-mat.str-el]. 
[6] R. E. Gamboa Saraví, G. L. Rossini, and F. A. Schaposnik, Int. J. Mod. Phys. A 11, 19962643.

[7] A. N. Redlich, Phys. Rev. Lett. 52 (1984) 18 ; Phys. Rev. D 29 (1984) 1984.

[8] G. V. Dunne, hep-th/9902115.

[9] S. Deser, L. Griguolo and D. Seminara, Phys. Rev. Lett. 79 (1997) 1976

[10] C. Fosco, G. L. Rossini and F. A. Schaposnik, Phys. Rev. Lett. 79 (1997) 1980.

[11] C. D. Fosco, G. L. Rossini and F. A. Schaposnik, Phys. Rev. D 56 (1997) 6547.

[12] G. V. Dunne, hep-th/9902115.

[13] S. R. Coleman, Annals Phys. 101, 239 (1976).

[14] S. Deser, L. Griguolo and D. Seminara, Phys. Rev. D 67 (2003) 065016.

[15] E. H. Fradkin and F. A. Schaposnik, Phys. Lett. B 338 (1994) 253

[16] J. C. Le Guillou, E. Moreno, C. Nunez and F. A. Schaposnik, Nucl. Phys. B 484 (1997) 682; Phys. Lett. B 409 (1997) 257.

[17] C. D. Fosco and F. A. Schaposnik, Phys. Lett. B 391 (1997) 136.

[18] E. F. Moreno and F. A. Schaposnik, Phys. Rev. D 88 (2013) no.2, 025033

[19] C. D. Fosco, E. F. Moreno and F. A. Schaposnik, Phys. Rev. D 85 (2012) 046005. 